\title{
MOTIONS OF ISOLATED $G$-BAND BRIGHT POINTS IN THE SOLAR PHOTOSPHERE
}

\author{
P. Nisenson and A. A. van Ballegooijen \\ Harvard-Smithsonian Center for Astrophysics, 60 Garden Street, MS 15, Cambridge, MA 02138; \\ pnisenson@cfa.harvard.edu, vanballe@cfa.harvard.edu \\ AND \\ A. G. DE WiJn AND P. SütTERLIN \\ Sterrekundig Instituut, P.O. Box 80,000, 3508 TA Utrecht, Netherlands; a.g.dewijn@astro.uu.nl, p.suetterlin@astro.uu.nl \\ Received 2002 November 20; accepted 2002 December 16
}

\begin{abstract}
Magnetic elements on the quiet Sun are buffeted by convective flows that cause lateral motions on timescales of minutes. The magnetic elements can be observed as bright points (BPs) in the $G$ band at $4305 \AA$. We present observations of BPs based on a long sequence of $G$-band images recorded with the Dutch Open Telescope and postprocessed using speckle-masking techniques. From these images we measured the proper motions of isolated BPs and derived the autocorrelation function of their velocity relative to the solar granulation pattern. The accuracy of BP position measurements is estimated to be less than $23 \mathrm{~km}$ on the Sun. The rms velocity of BPs (corrected for measurement errors) is about $0.89 \mathrm{~km} \mathrm{~s}^{-1}$, and the correlation time of BP motions is about $60 \mathrm{~s}$. This rms velocity is about 3 times the velocity measured using cork tracking, almost certainly due to the fact that isolated BPs move more rapidly than clusters of BPs. We also searched for evidence of vorticity in the motions of $G$-band BPs.
\end{abstract}

Subject headings: Sun: atmospheric motions — Sun: faculae, plages — Sun: magnetic fields techniques: high angular resolution

\section{INTRODUCTION}

Observations of the Sun with high spatial resolution show network bright points (Muller 1983, 1985, 1994; Keil \& Muller 1983; Muller \& Roudier 1984, 1992) and "filigree" (Dunn \& Zirker 1973; Mehltretter 1974; Berger et al. 1995), which are chains of bright features located within the intergranular lanes. The bright points and filigree are seen in the wings of strong spectral lines such as $\mathrm{H} \alpha$ and $\mathrm{Ca}$ II $\mathrm{H}$ and $\mathrm{K}$, in lines formed in the photosphere, and even at continuum wavelengths (with reduced contrast). The widths of these structures is $100-200 \mathrm{~km}$, at the limit of resolution of ground-based solar telescopes. In the following, we collectively refer to these bright structures as bright points (BPs). The BPs are associated with regions of strong magnetic field (Chapman \& Sheeley 1968; Title, Tarbell, \& Topka 1987; Simon et al. 1988; Title et al. 1989, 1992; Keller 1992) and correspond to magnetic flux tubes of kilogauss field strength that stand nearly vertically in the solar atmosphere (Stenflo 1973; Stenflo \& Harvey 1985; Sánchez Almeida \& Martínez Pillet 1994; see review by Solanki 1993).

The dynamical behavior of BPs has been studied by a number of authors. Muller (1983) found that facular points on the quiet Sun are predominantly located in patches at the periphery of supergranule cells, indicating that the magnetic elements are advected by the supergranular flow. The BPs always first appear in the dark spaces at the junction of several granules, never inside a granule or in the space between only two granules. As the granulation pattern evolves, the BPs remain in the intergranular spaces throughout their lifetime but not necessarily at the junction of several granules like at the time of their first appearance. New BPs have a tendency to appear adjacent to existing points, and $15 \%$ of the BPs seem to split into two points that move apart until a separation of $1^{\prime \prime}-1$ ". 5 is reached.
Muller et al. (1994) measured velocities of 29 isolated BPs and found a mean speed of $1.33 \mathrm{~km} \mathrm{~s}^{-1}$. Strous (1994) studied BPs in a growing active region. Using line-center images taken in $\mathrm{Fe}$ I $(5576 \AA)$, he found velocities between 0.26 and $0.62 \mathrm{~km} \mathrm{~s}^{-1}$. Berger \& Title (1996) measured velocities of $1-5 \mathrm{~km} \mathrm{~s}^{-1}$ for $G$-band (4305 $\mathrm{A}$ ) BPs in the "moat" around a sunspot; they showed that the motions are constrained to the intergranular lanes and are primarily driven by the evolution of the granulation pattern. They found that the BPs continually split up and merge, with a mean time between splitting events of few hundred seconds. Löfdahl et al. (1998) and Berger et al. (1998) analyzed G-band and continuum images obtained at the Swedish Vacuum Solar Telescope (SVST) on La Palma and found rapid splitting and merging of BPs in an enhanced network region. Van Ballegooijen et al. (1998) used a "cork-tracking" method to measure the proper motions of $G$-band BPs and found that BPs appear to be passively advected by the granulation flow. The filigree are known to be associated with abnormal granulation patterns (Dunn \& Zirker 1973), and the granules near network BPs are smaller and more numerous than near a normal intergranular space (Muller, Hulot, \& Roudier 1989), suggesting that the magnetic field has some effect on the granulation flow.

Little is known about the small-scale dynamics of flows in intergranular lanes and the interaction of magnetic elements with such flows. Theoretical models (e.g., Stein \& Nordlund 2000; Emonet \& Cattaneo 2001) predict that vorticity is concentrated in the lanes and that magnetic elements exhibit rapid rotational motions. At present, there is no direct observational evidence for vorticity in intergranular lanes. More detailed measurements of vorticity are needed for testing magnetoconvection models and as input for models of flux tube waves that heat the upper solar atmosphere (Hasan et al. 2003). 
In this paper we present measurements of BP proper motions within the intergranular lanes. In particular, we look for evidence of vortical motions. Tracking BPs is a difficult task. Long sequences of images with very good seeing (or corrections of seeing) are needed. Image jitter due to residual atmospheric effects needs to be reduced as much as possible. The measurements of BP positions need to be made with respect to some frame of reference. The solar granulation is used as a reference, and the motion of this reference frame is determined by correlation tracking on the granulation pattern. We also tested measuring the BPs with respect to the average position of the bright points in each field but found that the result is nearly identical to the result from correlation tracking. BPs vary widely in lifetime and contrast, so it is very difficult to automate the process of tracking them. In this paper we use manual selection of BPs followed by fitting the peaks.

\section{OBSERVATIONS}

Image sequences recorded with good seeing in the $G$ band $(4305 \AA)$ are essential for tracking BPs over many frames. Here we use $G$-band images recorded with the Dutch Open Telescope (DOT) (Hammerschlag \& Bettonvil 1998; Rutten et al. 2001, 2002; Sütterlin et al. 2001). The image sequence was collected on 2001 October 19, starting at 10:39 UT under good seeing conditions from a network region close to the disk center. The pixel size for these images was 0 ".071 (corresponding to $51.5 \mathrm{~km}$ on the Sun), and the filter bandpass was $10 \AA$. The data were recorded at a rate of 6 frames $\mathrm{s}^{-1}$, and a burst of 100 frames was collected every $30 \mathrm{~s}$. Each burst is combined into a single reconstructed frame using speckle image processing. The speckle processing uses an improved version of the code of de Boer, Kneer, \& Nesis (1992), who first applied the speckle-masking method of Weigelt (1977) to solar images, making use of the spectral line ratio technique (von der Lühe 1984). The resulting 199 frame sequence ( 1 frame every $30 \mathrm{~s}$ ) achieves 0 ."2 resolution over the entire sequence. This is very close to the diffraction limit of the $43.8 \mathrm{~cm}$ aperture. Figure 1 is a typical frame from the sequence.

The images in the sequence were crudely co-aligned by visual tracking of a group of BPs. We then selected two subregions, each $256 \times 256$ pixels in size, for measurement of BP positions (see Fig. 1). For each subregion, we used correlation tracking to determine a series of displacements of the granulation pattern in consecutive frames. These displacements were measured with subpixel accuracy by finding the peak of the cross-correlation of consecutive subimages and fitting a quadratic function to the values near the peak. We accumulated these displacements to obtain a series of positions of the granulation pattern, $\left(x_{\mathrm{gran}, n}, y_{\mathrm{gran}, n}\right)$, where $n$ is the frame index. We then examined each frame many times, using a cursor to select a BP and follow it for as many

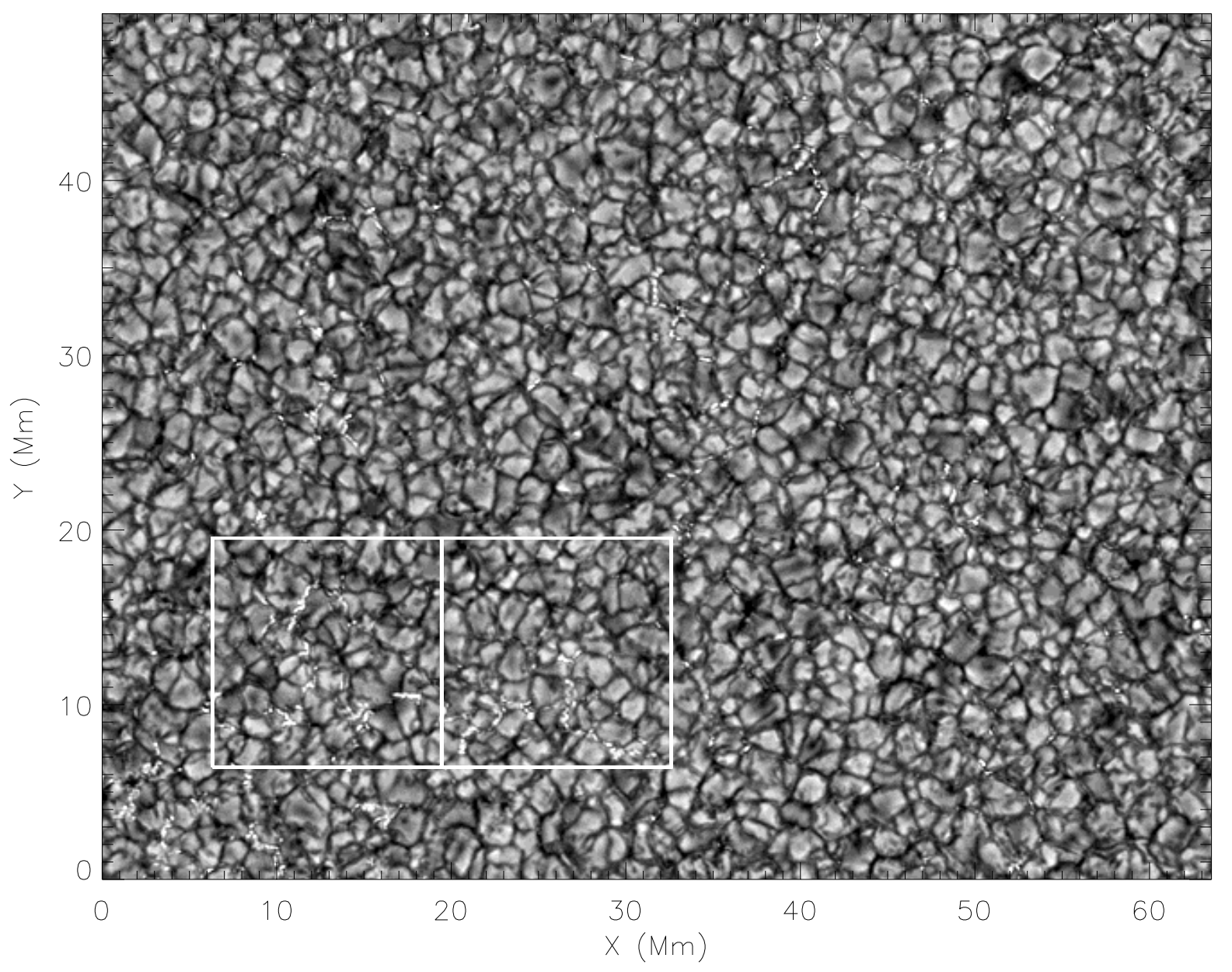

FIG. 1. $-G$-band frame taken at 10:39:35 UT. The boxes show the two subregions used for measurement of BP positions. 

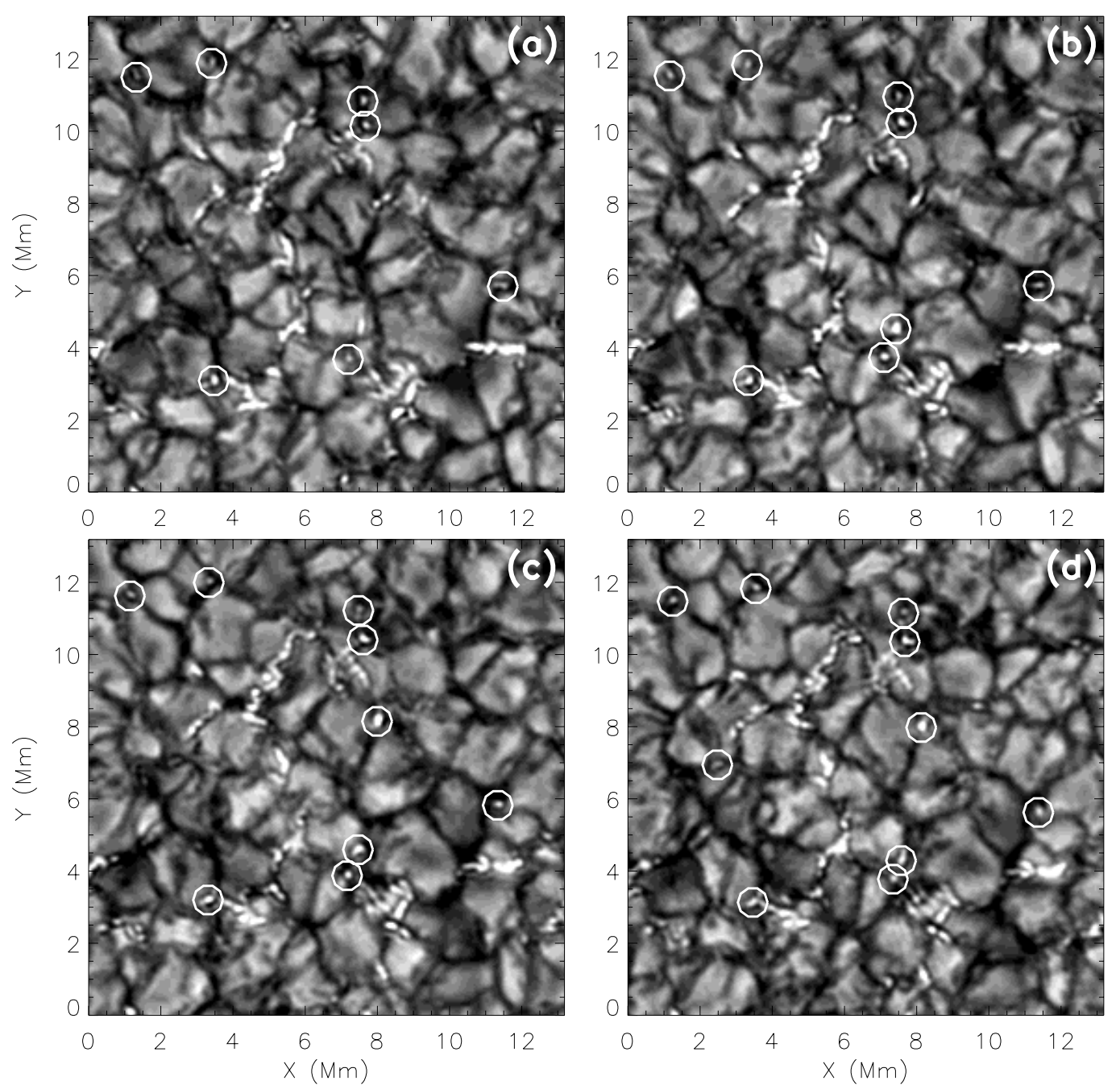

FIG. 2.-Four images and with BP locations circled. The frames are separated in time by 1 minute.

frames as we could, before it disappeared or merged with other BPs. Each BP position was again measured with subpixel accuracy by quadratic fitting, and the granulation position was subtracted. The result was a list of BP positions $x_{k, n}$ and $y_{k, n}$ in a reference frame tied to the solar granulation pattern, where $k$ is the BP index. The advantage of this method is that an accurate co-alignment and interpolation of the subimages is not required.

We only selected BPs that were clearly separated from other BPs so that we could follow them without confusing their identity. We stopped tracking them if they started to merge with other BPs. Any BPs that we could not track for at least 3.5 minute (7 frames) were discarded. Our measurements yielded 2992 positions for 161 BPs. The longest-living BP we were able to track lived for 25 minutes (50 frames). The mean lifetime was 9.2 minutes.

Figure 2 shows four subregion frames, separated in time by 1 minute. The circles show the positions of the BPs selected from each frame. Figure 3 shows the paths of four individual BPs. In some cases, like the top left and top right plots, the BP followed a simple curved path. In other cases, the motions were erratic and complicated, as in the bottom right plot. Some BPs move in nearly straight lines, following intergranular lanes.
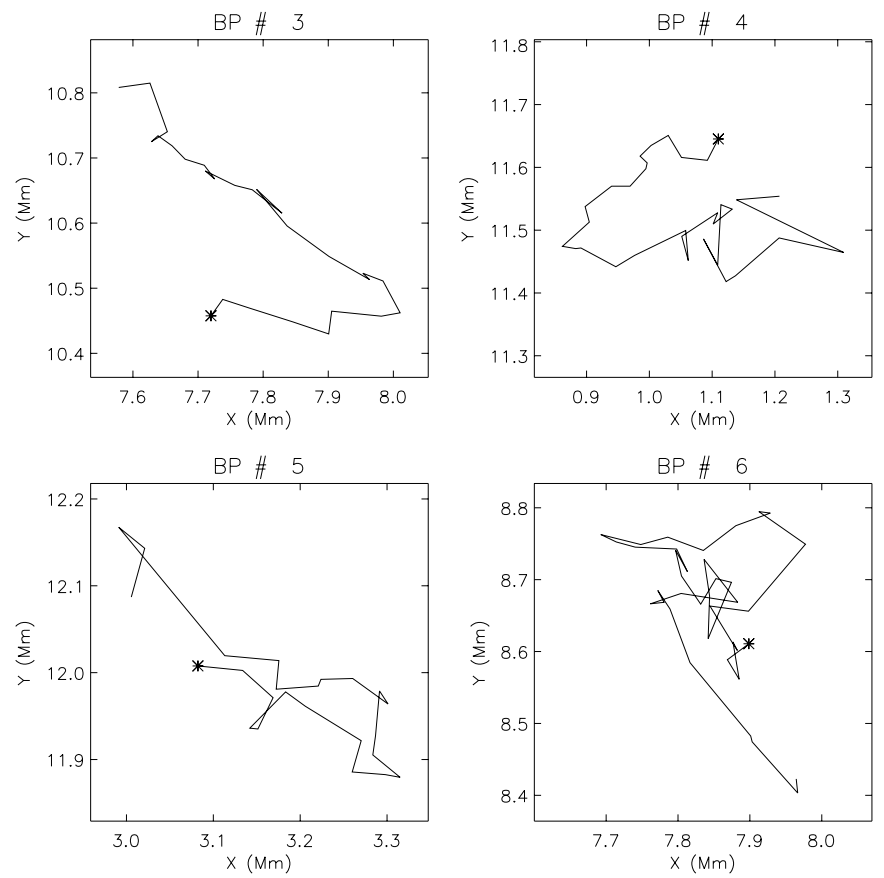

FIG. 3.-Four bright point tracks. The starting point of each track is indicated by a star. 


\section{ANALYSIS}

We calculated BP velocities $v_{x, k, n}$ and $v_{y, k, n}$ from position differences over time intervals of $30 \mathrm{~s}$. Figure 4 shows histograms of $v_{x}, v_{y}$, and $v\left[=\left(v_{x}^{2}+v_{y}^{2}\right)^{1 / 2}\right]$, and a scatter plot of $v_{x}$ versus $v_{y}$. The histograms are consistent with a Gaussian distribution with $\mathrm{rms}$ velocity of $1.31 \mathrm{~km} \mathrm{~s}^{-1}$. This includes both real signal and measurement errors. We also computed the autocorrelations $C_{x x, m}$ and $C_{y y, m}$ and the crosscorrelation $C_{x y, m}$,

$$
\begin{aligned}
& C_{x x, m}=\left\langle v_{x, k, n} v_{x, k, n+m}\right\rangle, \quad C_{y y, m}=\left\langle v_{y, k, n} v_{y, k, n+m}\right\rangle, \\
& C_{x y, m}=\left\langle v_{x, k, n} v_{y, k, n+m}\right\rangle,
\end{aligned}
$$

where $m$ is the number of time steps separating two velocity measurements for the same BP and the brackets denote an average over the BP index $k$ and frame number $n$. Figures $5 a$ and $5 b$ show the autocorrelations as function of the delay time $t=30 m$ (in units of seconds). Figure $5 c$ shows the cross-correlation function. The velocities $v_{x}$ and $v_{y}$ appear to be uncorrelated to within the measurement errors (note the expanded vertical scale of this figure compared to Figs. $5 a$ and $5 b$ ). Figure $5 d$ shows the number of measurements used to derive these correlation functions.

Each autocorrelation function shows a strong peak at zero time lag and dips at lags of \pm 1 frame ( $30 \mathrm{~s} \mathrm{time} \mathrm{delay).}$ We suggest that these features are due to the measurement errors. Let $u_{x, k, n}$ be the $x$-component of the true velocity of BP $k$ in the time interval between frames $n$ and $n+1$; then the measured velocity is

$$
v_{x, k, n}=u_{x, k, n}+\frac{\delta x_{k, n+1}-\delta x_{k, n}}{\Delta t},
$$

where $\Delta t=30(\mathrm{~s})$ is the time between frames and $\delta x_{k, n}$ are the errors in position measurement. Now assume that these errors are randomly distributed with standard deviation $\sigma$ (the same for all BPs) and are uncorrelated from frame to
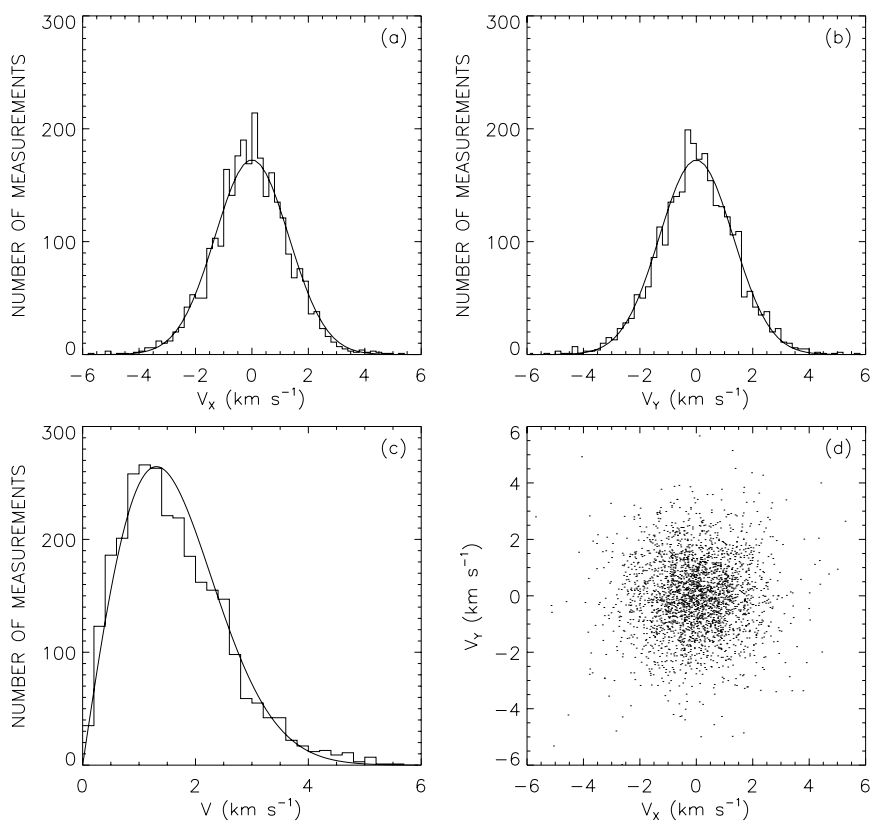

FIG. 4.-Histograms of measured BP velocities: $(a) v_{x},(b) v_{y}$, and $(c) v$ The results are compared with a Gaussian distribution with rms velocity $1.31 \mathrm{~km} \mathrm{~s}^{-1}$. (d) Shows $v_{x}$ plotted against $v_{y}$.
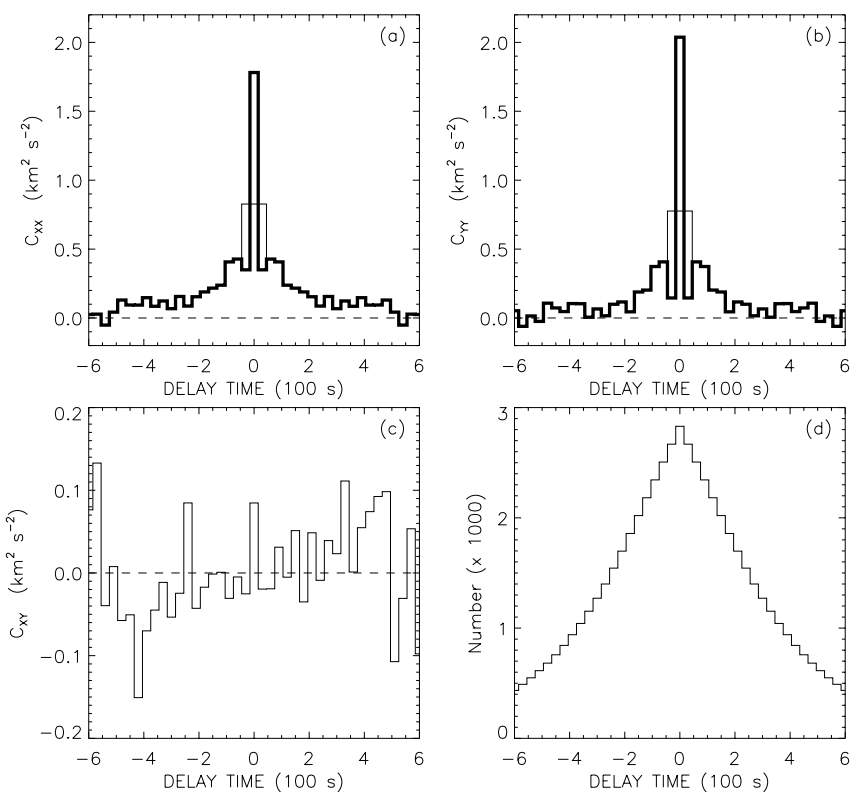

FIG. 5.-Correlation functions of BP velocity $v_{x}$ and $v_{y}$. (a) Observed autocorrelation $C_{x x, m}$ as function of delay time $t=30 \mathrm{~m}$ (in units of seconds) (thick curve) and the average autocorrelation $\tilde{C}_{x x}$ (thin curve). (b) Similar for the observed autocorrelation $C_{y y, m}$ (thick curve) and average correlation $\tilde{C}_{y y}$ (thin curve). (c) Cross-correlation $C_{x y, m}$ as function of delay time $t$. $(d)$ Number of measurements per bin used in panels $(a),(b)$, and $(c)$.

frame. Then the observed autocorrelation $C_{x x, m}$ is

$$
\begin{aligned}
C_{x x, 0} & =\left\langle u_{x, k, n}^{2}\right\rangle+\sigma_{v}^{2}, \\
C_{x x, \pm 1} & =\left\langle u_{x, k, n} u_{x, k, n \pm 1}\right\rangle-\sigma_{v}^{2} / 2, \\
C_{x x, m} & =\left\langle u_{x, k, n} u_{x, k, n+m}\right\rangle \text { for }|m| \geq 2,
\end{aligned}
$$

and similarly for $C_{y y, m}$, where $\sigma_{v}=\sigma \sqrt{2} / \Delta t$ is the error in the velocity measurement. The position errors increase the observed correlation at $m=0$ by $\sigma_{v}^{2}$ and reduce the correlation at $m= \pm 1$ by $-\sigma_{v}^{2} / 2$. Therefore, the average autocorrelations, defined by

$$
\tilde{C}_{x x} \equiv \frac{1}{3} \sum_{m=-1}^{+1} C_{x x, m}, \quad \tilde{C}_{y y} \equiv \frac{1}{3} \sum_{m=-1}^{+1} C_{y y, m},
$$

are not systematically affected by the measurement errors. These average correlations are indicated by the thin lines in Figures $5 a$ and $5 b$. The difference between $C_{x x, 0}$ and $\tilde{C}_{x x}$ provides an upper limit on $\sigma_{v}^{2}$ because the true autocorrelation $\left\langle u_{x, k, n} u_{x, k, n+m}\right\rangle$ decreases with increasing $|m|$. The autocorrelations for $v_{x}$ and $v_{y}$ yield upper limits on $\sigma_{v}$ of 0.98 and $1.12 \mathrm{~km} \mathrm{~s}^{-1}$, respectively. Therefore, the upper limit on the position error $\sigma$ is about $24 \mathrm{~km}$.

Figures $5 a$ and $5 b$ show that the autocorrelations are significantly different from zero for delays up to $500 \mathrm{~s}$. The average correlations are $\tilde{C}_{x x}=0.83 \pm 0.03$ and $\tilde{C}_{y y}=0.78$ $\pm 0.03 \mathrm{~km}^{2} \mathrm{~s}^{-2}$, where the error estimates are based on the cross-correlations shown in Figure 5c. This provides a lower limit on the true velocity dispersion $u_{0}$ of BPs in one direction, $u_{0} \approx 0.89 \mathrm{~km} \mathrm{~s}^{-1}$, based on the average of $\tilde{C}_{x x}$ and $\tilde{C}_{y y}$. Furthermore, $\tilde{C}_{x x}$ and $\tilde{C}_{y y}$ are about twice the autocorrelations at $m= \pm 2$. Therefore, the correlation time of the BP velocity is about $60 \mathrm{~s}$ (two frames).

We were interested in using BPs as a possible diagnostic of vortical motions in the intergranular lanes. A BP caught 

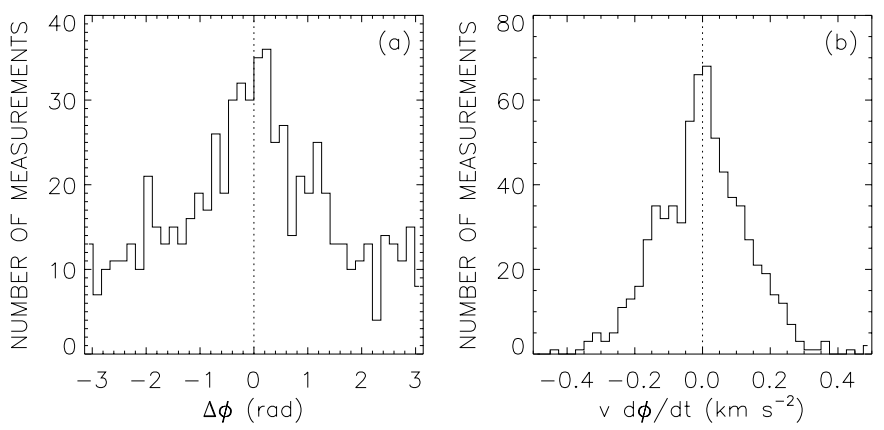

FIG. 6.-(a) Histogram of the change $\Delta \phi$ in direction angle of BP velocity. (b) Histogram of the centrifugal acceleration $v d \phi / d t$.

up in a vortex within an intergranular lane might exhibit rotational motions. In an effort to detect such motions, we computed changes in direction angle, $\Delta \phi$, of BP velocity in two neighboring time intervals. For this calculation, we discarded any measurements for which $v<1.5 \mathrm{~km} \mathrm{~s}^{-1}$ in either time interval, since the angle errors became large for smaller velocities. This left us with 687 measurements. A histogram of $\Delta \phi$ is shown in Figure $6 a$. The distribution shows a broad peak at $\Delta \phi=0$, indicating that the direction of motion persists from one time interval to the next. Figure $6 b$ shows a histogram of the centrifugal acceleration, $v d \phi / d t$, which is the relevant quantity when considering the generation of transverse waves in magnetic flux tubes. Histograms such as these could be useful tools for comparing models of flux tube waves to observations.

The above measurements use the granulation pattern as a reference frame. In order to check whether the granulation provides a stable reference frame, we also measured BP positions relative to each other (independent of the granulation pattern). For this purpose, we computed a reference position $\left(x_{\text {ref }, n}, y_{\text {ref }, n}\right)$ as follows. For each pair of frames $n$ and $n+1$, we determine the average positions $\left(\bar{x}_{n}, \bar{y}_{n}\right)$ and $\left(\bar{x}_{n+1}, \bar{y}_{n+1}\right)$ of all BPs in common between these consecutive frames. Then we accumulate the position differences

$$
\begin{aligned}
& x_{\text {ref }, n+1}=x_{\text {ref }, n}+\bar{x}_{n+1}-\bar{x}_{n}, \\
& y_{\text {ref }, n+1}=y_{\text {ref }, n}+\bar{y}_{n+1}-\bar{y}_{n},
\end{aligned}
$$

starting with $x_{\text {ref }, 0}=y_{\text {ref }, 0}=0$ in the first frame. This provides a reference position against which the individual BPs can be measured. The relative positions are $x_{k, n}^{\prime}=$ $x_{k, n}-x_{\text {ref }, n}$ and $y_{k, n}^{\prime}=y_{k, n}-y_{\text {ref }, n}$. The autocorrelations of $v_{x}^{\prime}$ and $v_{y}^{\prime}$ yield $C_{x x}=0.71 \pm 0.03$ and $\tilde{C}_{y y}=$ $0.66 \pm 0.03 \mathrm{~km}^{2} \mathrm{~s}^{-2}$, which is about $15 \%$ less than the values derived for the granulation reference frame. This reduction in BP velocities is to be expected because there are on average only $7.0 \mathrm{BPs}$ in common between consecutive frames, and subtracting the mean velocity of these 7 BPs should reduce $\tilde{C}_{x x}$ and $C_{y y}$ by a factor of $\frac{6}{7}$. The fact that the observed reduction is not any larger than predicted implies that there is nothing to be gained by measuring the $\mathrm{BP}$ motions relative to each other, and in fact, this causes the $\mathrm{BP}$ velocities to be systematically underestimated. We conclude that the granulation pattern provides a stable reference frame for measurement of BP motions.

\section{DISCUSSION}

We have shown that with good enough data we can precisely measure positions for isolated BPs, obtaining both their linear and angular motions. The errors due to image jitter have been reduced to levels of $25 \mathrm{~km}$ ( 0.5 pixels) or less. This allows us to measure the motions of individual BPs to a precision of less than $1 \mathrm{~km} \mathrm{~s}^{-1}$ for each $30 \mathrm{~s}$ time interval, which will be important for studying the generation of MHD waves in flux tubes. We also track BPs for sufficiently long times that we can see the regularity in their motions.

The rms velocities we measured for isolated BPs were almost a factor of 3 higher than velocities measured using cork tracking (van Ballegooijen et al. 1998). We believe this is due to a selection effect. By measuring only isolated BPs, we measure the fastest-moving features. Once BPs cluster with other BPs, mostly at lane interstices, they seem to be "captured" by the group and their motions are reduced. There are many more BPs in these clusters than there are isolated BPs so the cluster statistics dominate when measurements are made with cork tracking and the average velocity is reduced.

In measuring the motions of BPs, we have found that they move in somewhat circular paths. Combining the angular change of their motions with the distance they travel is a potential way of estimating the (vertical) vorticity in the flow field, assuming the BPs act as test particles. While performing the tracking of individual BPs, we saw little evidence of vorticity other than the tracks of the BPs themselves. We looked for, but did not find, pairs of BPs that orbited one another; in fact, BPs that came in close proximity to other BPs tended to have reduced motion. We also saw little evidence of swirling motions in the granules or granular lanes that would correspond to the observed BP motions. This might indicate that BPs are affected not only by surface flows associated with the solar granulation but also by other flows that occur at larger depth below the photosphere. Further modeling will be required to establish whether the BPs move passively with the surface flow and to determine whether the observed BP motions are consistent with models of flux tube waves and heating of the upper solar atmosphere.

The authors wish to thank R. J. Rutten, R. Hammerschlag, and F. Bettonvil for the use of DOT solar data. We also wish to especially thank R. J. Rutten for his very helpful suggestions on an earlier draft of this paper. We wish to acknowledge support for the work from the National Science Foundation (grant ATM-9811523) to the Smithsonian Astrophysical Observatory.

\section{REFERENCES}

Berger, T. E., Löfdahl, M. G., Shine, R. A., \& Title, A. M. 1998, ApJ, 495 , 973

Dunn, R. B., \& Zirker, J. B. 1973, Sol. Phys., 33, 281

Emonet, T., \& Cattaneo, F. 2001, ApJ, 560, L197

Hammerschlag, R. H., \& Bettonvil, F. C. M. 1998, NewA Rev., 42, 485

Hasan, S. S., Kalkofen, W., van Ballegooijen, A. A., \& Ulmschneider, P. 2003, ApJ, 585, 1138

Scharmer, G. 1995, ApJ, 454, 531

Berger, T. E., \& Title, A. M. 1996, ApJ, 463, 365

Chapman, G. A., \& Sheeley, N. R., Jr. 1968, Sol. Phys., 5, 442

de Boer, C. R., Kneer, F., \& Nesis, A. 1992, A\&A, 257, L4

Keil, S. L., \& Muller, R. 1983, Sol. Phys., 87, 243

Keller, C. U. 1992, Nature, 359, 307 
Löfdahl, M. G., Berger, T. E., Shine, R. A., \& Title, A. M. 1998, ApJ, 495, 965

Mehltretter, J. P. 1974, Sol. Phys., 38, 43

Muller, R. 1983, Sol. Phys., 85, 113 1985, Sol. Phys., 100, 237

1994, in Solar Surface Magnetism, ed. R. J. Rutten \& C. J. Schrijver (Dordrecht: Kluwer), 55

Muller, R., Hulot, J. C., \& Roudier, Th. 1989, Sol. Phys., 119, 229

Muller, R., \& Roudier, Th. 1984, Sol. Phys., 94, 33 1992, Sol. Phys., 141, 27

Muller, R., Roudier, Th., Vigneau, J., \& Auffret, H. 1994, A\&A, 283, 232

Rutten, R. J., Hammerschlag, R. H., Sütterlin, P., \& Bettonvil, F. C. M. 2001, in ASP Conf. Ser. 236, Advanced Solar Polarimetry: Theory, Observation, and Instrumentation, ed. M. Sigwarth (San Francisco: ASP), 25

Rutten, R. J., Sütterlin, P., de Wijn, A. G., Hammerschlag, R. H., Bettonvil, F. C. M., Hoogendoorn, P. W., \& Jägers, A. P. L. 2002, in Proc. 10th European Solar Physics Meeting, Solar Variability, From Core to Outer Frontiers, ed. M. Carlsson \& P. Ambroz (ESA SP-506; S.I.: EPS), 903

Sánchez Almeida, J., \& Martínez Pillet, V. 1994, in Solar Magnetic Fields, ed. M. Schüssler \& W. Schmidt (Cambridge: Cambridge Univ. Press), 316
Simon, G. W., Title, A. M., Topka, K. P., Tarbell, T. D., Shine, R. A., Ferguson, S. H., Zirin, H., \& the SOUP Team. 1988, ApJ, 327, 964

Solanki, S. 1993, Space Sci. Rev., 63, 1

Stein, R. F., \& Nordlund, A. 2000, Sol. Phys., 192, 91

Stenflo, J. O. 1973, Sol. Phys., 32, 41

Stenflo, J. O., \& Harvey, J. W. 1985, Sol. Phys., 95, 99

Strous, L. 1994, in Solar Surface Magnetism, ed. R. J. Rutten \& C. J. Schrijver (Dordrecht: Kluwer), 73

Sütterlin, P., Hammerschlag, R. H., Bettonvil, F. C. M., Rutten, R. J., Skomorovsky, V. I., \& Domyshev, G. N. 2001, in ASP Conf. Ser. 236 Advanced Solar Polarimetry: Theory, Observation, and Instrumentation, ed. M. Sigwarth (San Francisco: ASP), 431

Title, A. M., Tarbell, T. D., \& Topka, K. P. 1987, ApJ, 317, 892

Title, A. M., Tarbell, T. D., Topka, K. P., Ferguson, S. H., Shine, R. A., \& the SOUP Team. 1989, ApJ, 336, 475

Title, A. M., Topka, K. P., Tarbell, T. D., Schmidt, W., Balke, C., \& Scharmer, G. 1992, ApJ, 393, 782

van Ballegooijen, A. A., Nisenson, P., Noyes, R. W., Löfdahl, M. G., Stein,

R. F., Nordlund, A., \& Krishnakumar, V. 1998, ApJ, 509, 435

von der Lühe, O. 1984, J. Opt. Soc. Am., 1, 510

Weigelt, G. P. 1977, Opt. Commun., 21, 55 